\title{
Chapter 26 \\ Prospects for Liposome-Encapsulated Nisin in the Prevention of Dental Caries
}

\author{
Hideaki Tsumori, Yoshitaka Shimizu, Kohei Nagatoshi, Yutaka Sakurai, \\ and Kazuo Yamakami
}

\begin{abstract}
Dental caries is a common oral bacterial infectious disease. Its prevention requires the control of the causative pathogens, such as Streptococcus mutans, that exist within dental plaque. Nisin is a proteinaceous bacteriocin produced by Lactococcus lactis that is used to suppress bacterial infections. It has an inhibitory mode of action on a wide range of gram-positive bacteria. Improvements in the medical benefits of antibacterial agents can be achieved if they can be retained in liposomes for a long period after administration. Liposome systems can increase the ability of the encapsulated compounds, which have been widely used to encapsulate many kinds of compounds in various scientific settings. Liposomes can release labile molecules at a moderate rate. Liposome technologies that effectively protect the encapsulated molecules from decomposition have the potential to improve their preventive and therapeutic effects. Therefore, the use of liposomes to administer antimicrobial agents has spurred research into their utility in preventive medicine. The encapsulation of nisin in liposomes can provide means of improving the stability of nisin and its antibacterial effect against $S$. mutans. The present chapter will review the prospects for liposome-encapsulated nisin for the prevention of oral infectious diseases.
\end{abstract}

Keywords Dental caries $\bullet$ Liposome $\bullet$ Nisin $\bullet$ Preventive medicine $\bullet$ Streptococcus mutans

\footnotetext{
H. Tsumori

Department of Chemistry, National Defense Medical College, Tokorozawa 359-8513, Japan

e-mail: htsumori@ndmc.ac.jp

Y. Shimizu

Research and Development Division, BioMedCore Inc., Sapporo 001-0021, Japan

e-mail: shimizuy@bmcore.co.jp

K. Nagatoshi

Eco Friendly Institute Ltd., Fukuoka 818-0013, Japan

e-mail: kona_bj5pp1@yahoo.co.jp

Y. Sakurai $\bullet$ K. Yamakami $(\bowtie)$

Department of Preventive Medicine and Public Health, National Defense Medical College,

3-2 Namiki, Tokorozawa, Saitama 359-8513, Japan

e-mail: ysakurai@ndmc.ac.jp; yamakami@ndmc.ac.jp
} 


\subsection{Introduction}

Oral diseases related to cariogenic microorganisms, such as dental caries, affect majority of the world's populations [1]. Dental caries results from interaction of specific bacteria with dietary carbohydrates in the oral cavity. Streptococcus mutans is a key contributor to the formation of cariogenic glucan biofilms. Nisin is an effective bactericidal agent against gram-positive bacteria [2]. It is a bacteriocin with a molecular mass of 3,354 and is produced by certain strains of Lactococcus lactis subsp. lactis [3]. The bactericidal activity of nisin against cariogenic streptococci was investigated in vitro and the findings indicated that nisin acts as an inhibitor of typical cariogenic streptococci [4, 5]. It may be possible to use liposomes as a carrier for the effective administration of nisin. Liposomes are artificial spherical vesicles that can be created from lipids. They have been developed and evaluated as carriers to deliver encapsulated molecules to target organs and specific cells in vitro and in vivo [6-9]. Investigations on liposomes have led to their use with many substances, and the encapsulated agents are pharmacodynamically and pharmacokinetically much more efficient than the naked compounds. Liposome-encapsulated substances have been used in various applications such as to retain and control the release of antibacterial compounds [10, 11]. They have been used to deliver pharmaceuticals, and their recent application in the preservation of foods by the encapsulation of nisin has spurred further research into their use with food materials $[12,13]$. With regard to liposome formulations, a variety of factors, such as lipid composition, cholesterol content, the presence of a charge on the lipid bilayer, and the size of the vesicle, affect the effectiveness of liposomes as preventive and therapeutic agents [6]. Oral delivery of liposomal bacteriocins is a useful for the treatment to prevent dental caries [5]. Encapsulation technologies, which may shield substances such as nisin from degradation by digestive enzymes, and effectively deliver the encapsulated contents at the same time, could be an advancement in the field of preventive medicine [10].

This review article provides brief background information on liposomal nisin and explores its possible applications with a focus on factors affecting its effectiveness and the advantages it offers for human health by preventing dental caries.

\subsection{Cariogenic Microorganisms and Dental Caries}

The oral cavity harbors many microorganisms that together constitute a complex micro-ecological environment [14]. Dental caries, a chronic infectious disease, is induced by cariogenic microorganisms such as Streptococcus spp. The key virulence factors of the microorganisms, insoluble glucans, can be identified when they colonize dental plaque on the surface of the teeth $[4,15]$. S. mutans in particular has been recognized as a decisive factor in dental caries. Insoluble glucans provide specific binding sites for oral bacterial colonization of the tooth surface and confer 
structural integrity on the extracellular matrix, and therefore they are essential for the formation and accumulation of dental glucan biofilms [16]. Because insoluble glucans are synthesized by glucosyltransferases of $S$. mutans and these glucans provide a matrix for dental plaque biofilm, the streptococcus is considered to contribute to the formation of cariogenic biofilms [16, 17]. The streptococcus tenaciously adheres to the glucan and is highly acidgenic and acid-tolerant; these are critical virulence properties in the pathogenesis of dental caries [18]. Therefore, dental caries results from the interaction of $S$. mutans with dietary carbohydrates in the oral cavity; the formation of insoluble glucan biofilms on the tooth surface is a key result of the diet-bacterium interaction [19].

\subsection{Nisin as a Tool for Preventive Medicine}

Nisin is approved for use in foods and is employed as a food preservative in more than 50 countries [20]. It belongs to a group of bactericidal peptides called type A lantibiotics [21]. Two main natural variants of nisin, nisin $\mathrm{A}$ and nisin $\mathrm{Z}$, have been discovered [3]. Nisin $\mathrm{Z}$ is widely distributed and contains asparagine instead of histidine at position 27 of the amino acid sequence of nisin A. The two variants have nearly equal bactericidal activity, membrane insertion, and pore-forming ability. Nisin is bactericidal against a broad range of gram-positive microorganisms, such as Staphylococcus aureus and Listeria monocytogenes, and prevents the outgrowth of many Clostridium spp. and Bacillus spp. [11, 13]. However, stability issues such as proteolytic degradation and oxidation result in reduced bioactivity. It is soluble and stable at acidic $\mathrm{pH}$, but at alkaline $\mathrm{pH}$ values its solubility decreases and it becomes biologically inactive [22]. Nisin is a peptide composed of 34 amino acids, including one lanthionine, four $\beta$-methyl-lanthionine, one dehydrobutyrine, and two dehydroalanine residues. The internal thioester rings formed by lanthionine are responsible for the conformation of nisin [23]. The lanthionine rings act as conserved binding motifs for the recognition of specific targets and create segments of defined spatial structures in the nisin molecule [24]. The bactericidal activity of nisin has been suggested to be a result of electrostatic interaction of the positively charged carboxylterminal end of the molecule with negatively charged bacterial membrane lipids [25].

Nisin antagonizes a broad spectrum of gram-positive bacteria [26, 27]. It has dual activity against spore-forming bacteria, it inhibiting the outgrowth of spores and killing cells in the vegetative state. The didehydro amino acid residues in nisin interact with the membrane sulfhydryl groups of germinating spores [26]. Membrane disruption is considered to be the result of incorporation of nisin into the membrane and subsequent ion channel or pore formation [25]. Membrane potential is abolished in sensitive gram-positive cells as a result of the efflux of $\mathrm{K}^{+}$, amino acids and ATP through the membrane pores [28]. Subsequent leakage of ions causes catastrophic changes in transmembrane potential and internal pH. Nisin interacts with high-affinity pyrophosphate binding sites on the membrane-bound 
cell wall precursor Lipid II, leading to more effective formation of pores and inhibition of cell wall peptidoglycan biosynthesis [25, 29]. From these investigations, it is evident that nisin should be an effective antibacterial agent $[30,31]$.

\subsection{Nisin Against Cariogenic Streptococci}

Some antimicrobial agents, such as chlorohexidine, triclosan, xylitol, and cetylpyridinium chloride, have attracted interest as agents for prophylaxis against dental caries by inhibiting the multiplication of cariogenic microorganisms in the oral cavity [4]. However, these agents may cause some side effects such as discoloration of the teeth and tongue, drug resistance, and low solubility [4]. Because nisin is a lanthibiotic, unlike antibiotics, it does not produce drug resistance or chiasmatic resistance [21]. The bactericidal ability of nisin against cariogenic streptococci, such as $S$. mutans, has been investigated as a means of preventing dental caries [4]. Findings suggest that nisin has a potential to be used as a bactericidal agent to prevent dental caries. Liposomal nisin was shown to inhibit S. mutans [5]. In addition, nisin did not appear to be toxic to normal human gingival fibroblast and epithelial cells [32]. Therefore, nisin plays a role in inhibiting the viability of cariogenic streptococci.

\subsection{Liposomes as a Tool for Preventive Medicine}

Liposomes are formulated from phospholipid bilayers and consist of colloidal dispersions of lipids in aqueous buffers [33, 34]. Their formation is based on the interactions between phospholipids and water molecules in which the polar headgroups of phospholipids are exposed to the inner and outer aqueous phases and the hydrophobic carbohydrate tails are forced to face each other in a bilayer $[12,35]$. Liposomal encapsulation has been shown to stabilize the encapsulated compound against enzymatic degradation and chemical modification [36]. Liposomes can encapsulate hydrophobic and hydrophilic molecules, prevent the decomposition of the encapsulated molecules, and release the compound at designated target organs $[37,38]$. The bioactivity of encapsulated molecules can be maintained by the phospholipid vesicle until it is delivered to the target organ or cells, where the contents will be released [9, 39]. Liposomes can range in size from $40-50 \mathrm{~nm}$ to $1-2 \mu \mathrm{m}$, depending on the method of formulation, lipid components, and intended use [40]. One of the aims of liposome encapsulation in medicine is to increase the therapeutic index of the bioactive material [36, 41]. Encapsulation techniques have been developed in many fields because of the biocompatibility of liposomes, i.e., its ability to encapsulate both hydrophilic and lipophilic molecules, and its ability to deliver these molecules to target sites [42, 43]. In the food industry, liposomes have been used to deliver antibiotics, peptides, vitamins, and 
flavors $[44,45]$. The encapsulation of bacteriocins, such as nisin in liposomes represents an alternative to overcome problems related to the exploitation of bactericidal activities [5, 40]. Liposomes have been developed and used as carriers for therapeutic agents to improve the delivery of many anticancer and antibiotic compounds [46-48].

The usefulness of conservative therapeutics is restricted by their inability to deliver compounds to target sites. Attempts have been made to overcome this difficulty by providing selective delivery and release of encapsulated compounds. Hydrophobic compounds can be directly encapsulated into liposomes during vesicle formation, and retention is governed by interactions between the compound and lipid. Passive encapsulation of soluble compounds depends on the ability of liposomes to trap aqueous buffer containing dissolved compounds during vesicle formation [49]. The applications of liposomes in medicine and pharmacology can be divided into therapeutic and preventive uses of liposomes composed of various substances $[9,50,51]$. Advances in liposome design lead to applications for the delivery of new technology products such as nucleotides involved in numerous biological functions $[48,52]$. There have been many studies on the viability of a range of conservative chemicals formulated in liposomes, frequently resulting in improved biological activity compared with that of the naked form. Many antibiotics are orally available and liposome encapsulation can be developed for labile chemicals with minimal loss of utility. Therefore, liposomes are used in pharmaceutical applications and the encapsulated substances have enhanced efficacy compared with the naked substances. Liposomes are achieving medical acceptance because of the effective shelf life of labile molecules, slow and steady release of the molecules, and delivery of the molecules to required locations [46]. In pharmaceutical applications, liposomes offer drug delivery systems for therapeutic and preventive strategies [53].

\subsection{Characteristics of Liposomes}

The design of liposomal delivery systems accelerates the treatment on human health [6]. The strategy for liposomes is the development of carrier systems with the ability to deliver and release encapsulated compounds [54]. The characteristics of liposomes in this regard depend on their lipid composition, surface charge, and vesicular size. Because bilayer fluidity and rigidity can affect the release of liposomal compounds, studies have been carried out on the effects of phospholipids of different phase transition temperatures on fluidity and the effects of lipid composition on rigidity of liposomes $[55,56]$. Liposomes with many different lipid compositions exist, and those that include phospholipids provide efficient vesicles for individual applications [57].

The aim of lipid selection for liposomes is to provide stabilizing and encapsulation abilities. Phosphatidylcholines have been selected for the preparation of liposome vesicles in many settings. The amount of encapsulated content released 
from vesicles is proportional to the acyl chain length of the phosphatidylcholine. Dimyristoylphosphatidylcholine (DMPC), dipalmitoylphosphatidylcholine (DPPC) and distearoylphosphatidylcholine (DSPC) have longer saturated acyl chains (14, 16, and 18 carbons, respectively) and their phase transition temperature increases with increasing chain length [58]. The trend in the release of encapsulated compounds from vesicles is in the order of DMPC $>$ DPPC $>$ DSPC, which reflects the fluidity of vesicles composed of DMPC, DPPC, and DSPC with a phase transition temperature of 23,41 , and $55{ }^{\circ} \mathrm{C}$ respectively [57]. For example, the configuration of DPPC is a highly ordered gel phase at room temperature; however, above $41{ }^{\circ} \mathrm{C}$ the lipid forms a liquid crystalline phase characterized by greater packing disorder because of temperature-induced changes in acyl chain composition [58]. Unsaturated phospholipid species show more permeable and fluidic bilayers, but saturated phospholipids with long acyl chains form a rigid, rather impermeable bilayer structure $[33,37]$.

The cholesterol in phosphatidylcholine-based liposomes can reduce liposome permeability, because membrane permeability is dependent on fluidity and rigidity [59]. Cholesterol interacts with fatty acids in liposomes by hydrogen bonding, increasing the cohesiveness and mechanical strength of the vesicular membrane [60]. For example, a comparison of nisin-containing phosphatidylcholine liposomes and phosphatidylcholine plus cholesterol showed that cholesterol reduced the release of nisin $[38,61]$. The permeability of liposomes can be altered by modifying the cholesterol concentration according to the intended application of the liposomes [57, 62]. Sphingomyelin also increases the rigidity of the bilayer [6]. Therefore, the modification of liposomal composition enables the preparation of preferred kinds of liposomes for various scientific settings.

The effect of charge on lipid behavior is governed by the surface charge density of the liposomes, lipid head groups, and interactions between the encapsulated content and lipid [63, 64]. Liposomes composed of charged polar lipids with higher electrical charges are more stable than those composed of neutral polar lipids. A surface charge on liposomes increases repulsive interactions and reduces the frequency of liposome collisions [65]. With regard to control of the release of encapsulated compounds from liposomes, the charge has been observed to affect vesicle permeability [63]. The use of negatively charged liposomes containing phosphatidylglycerol led to greater effectiveness of the encapsulated molecules compared with the use of neutral or positively charged liposomes. Therefore, potency of the release of encapsulated compounds has been shown to be regulated by polymer size, lipid composition, and surface charge [65].

Liposomes can be used as carriers of many different kinds of compound, such as peptides and nucleotides, because liposomes are spherical vesicles whose membranes are composed of one or more phospholipid bilayers [66]. Phospholipid vesicles are capable of encapsulating labile molecules, and the lipids that they are composed are biodegradable and safe in vivo [67]. Liposome encapsulation of therapeutic and cosmetic agents can extend their activity by improving their stability and permeability and by providing targeted and timed release [68]. Labile compounds that are susceptible to proteolytic degradation, such as insulin, 
calcitonin, parathyroid hormone, and erythropoietin, were shown to have improved pharmacological effects after oral administration when encapsulated in liposomes [69]. Oral delivery of liposomal compounds is useful for preventive and therapeutic treatments.

\subsection{Characteristics of Liposome-Encapsulated Nisin}

Nisin is a cationic, amphiphilic antimicrobial peptide that inhibits gram-positive bacteria [30]. The encapsulation efficacy of nisin is higher in liposomes composed of neutral zwitterionic phospholipids, such as phosphatidylcholine, compared with liposomes composed of anionic phospholipids, such as phosphatidylglycerol $[40,61]$. The highest nisin encapsulation efficacy was achieved in liposomes having low contents of negatively charged phospholipids and high contents of zwitterionic phospholipids [70]. Encapsulation of positively charged nisin in anionic phospholipids should result in association because of attractive electrostatic interactions, whereas encapsulation in neutral liposomes should result in association because of hydrophobic interactions [71]. The electrostatic interaction of nisin with negatively charged membrane phospholipids is more pronounced than its interaction with neutral phospholipids [25]. Cationic vesicles containing stearylamine showed lower encapsulation efficiency when compared with other kind of vesicles, which could result from electrostatic repulsion between positively charged nisin and cationic vesicles [35]. The functional properties of liposomal nisin depend on the interaction of nisin with the liposome membrane and with the bacterial cell membrane. Such electrostatic interactions are considered to be the initial step in the series of events leading to membrane pore formation [72]. Nisin proved to have high penetration ability for anionic phospholipids and low penetration ability for neutral phospholipids [71]. Nisin in liposomes composed of phosphatidylcholine demonstrated the slowest release of nisin, whereas liposomes composed of phosphatidylcholine plus phosphatidylglycerol appeared to release efficiently [65]. Because nisin is positively charged at neutral $\mathrm{pH}$, electrostatic interaction with negatively charged phosphatidylglycerol was attractive and should have led to the formation of unstable pores because of binding to the charged phospholipid head groups of phosphatidylglycerol [25]. The activity of the encapsulated nisin is expressed by providing relatively short-term effects by release of the encapsulated nisin and long-term effects by deposition of lipid-membrane-immobilized nisin, i.e., bactericidal activity [28]. Therefore, encapsulated nisin and membraneimmobilized nisin provide a tool for inhibiting pathogenic microbes.

Regarding the applications of nisin, various studies have shown the efficiency of liposome-encapsulated nisin in food models [12]. The encapsulation of nisin improves its antimicrobial activity as a food preservative and for the prevention of oral infectious diseases [73]. Liposomal nisin should be able to provide effective long-term inhibition of the target microbe, S. mutans, and therefore preventing dental caries [5]. The surface charge of the liposome influences the interaction 
between liposomal nisin and bacteria [63]. As the bacterial cell has a negative charge, electrostatic repulsion is induced between the cell surface and liposomes composed of phosphatidylglycerol, preventing direct contact of the liposome with the microbes, and the subsequent release of nisin [61]. This assumption supports the idea that interaction between liposomes and microbes improves membrane fusion [73].

\subsection{Liposome-Encapsulated Nisin as a Preventive Agent for Oral and Dental Health}

There are relatively few published data regarding liposome-encapsulated nisin for the prevention of oral infectious diseases. There is a report that liposomal nisin inhibited the viability of S. mutans in vitro [5]. The inhibition of streptococcal viability led to the suppression of insoluble glucan formation by the streptococcus [5]. Previous studies have reported the synergistic antibacterial effects of combinations of antimicrobials, such as nisin plus ethylenediaminetetraacetic acid (EDTA), against gram-negative pathogens [74]. The ability of EDTA to destabilize the outer membrane of gram-negative bacteria by sequestering ions such as $\mathrm{Ca}^{2+}$ and $\mathrm{Mg}^{2+}$ is considered to enable nisin to access the cell membrane, thereby increasing its inhibitory effect [74]. The feacibility of encapsulating nisin plus EDTA in vesicles for the inhibition of L. monocytogenes and Escherichia coli O157:H7 was investigated $[43,65]$. Coencapsulation of nisin plus EDTA increased the inhibitory potential of liposomes against gram-positive S. mutans and gramnegative Porphyromonas gingivalis [40, 74]. These findings indicate that the liposomal nisin plus EDTA can inhibit the both of which could prevent dental caries and periodontitis.

\subsection{Prospects for Liposome-Encapsulated Nisin}

Encapsulation of nisin in liposome carrier provides long-lasting inhibition of the oral pathogen $S$. mutans. The appropriate balancing of the lipid components of the vesicles allows the construction of stable liposomal nisin that provides predictable release of the bactericidal agent. The results obtained so far encourage the study of liposomal nisin with the aim of developing potential tools for the prevention of dental caries. We are focusing on the application of liposomal technology in order to ascertain the bactericidal potential of liposomes as an effective carrier of nisin for oral health in humans.

Open Access This chapter is distributed under the terms of the Creative Commons Attribution Noncommercial License, which permits any noncommercial use, distribution, and reproduction in any medium, provided the original author(s) and source are credited. 


\section{References}

1. Marsh PD. Are dental diseases examples of ecological catastrophe? Microbiology. 2003;149:279-94.

2. Field D, Connor PMO, Cotter PD, Hill C, Ross RP. The generation of nisin variants with enhanced activity against specific gram-positive pathogens. Mol Microbiol. 2008;69:218-30.

3. Suganthi V, Selvarajan E, Subathradevi C, Mohanasrinvasan V. Lantibiotic nisin: natural preservative from Lactococcus lactis. Int Res J Pharm. 2012;3:13-9.

4. Tong Z, Dong L, Zhou L, Tao R, Ni L. Nisin inhibits dental caries-associated microorganism in vitro. Peptides. 2010;31:2003-8.

5. Yamakami K, Tsumori H, Sakurai Y, Shimizu Y, Nagatoshi K, Sonomoto K. Sustainable inhibition efficacy of liposome-encapsulated nisin insoluble glucan-biofilm synthesis by Streptococcus mutans. Pharm Biol. 2013;51:267-70.

6. Katragadda A, Bridgman R, Betageri G. Effect of liposome composition and cholesterol on the cellular uptake of stavudine by human monocyte/macrophages. Cell Mol Biol Lett. 2000;5:483-93.

7. Jia Y, Joly H. Liposome as a carrier for gentamycin delivery: development and evaluation of the physicochemical properties. Int J Pharm. 2008;359:254-63.

8. Mozafari MR, Pardakhty A, Azarmi S, Jazayeri JA, Nokhodchi A, Omri A. Role of nanocarrier systems in cancer nanotherapy. J Liposome Res. 2009;19:310-21.

9. Shimizu Y, Takagi H, Nakayama T, Yamakami K, Tadakuma T, Yokoyama N, et al. Intraperitoneal immunization with oligomannose-coated liposome-entrapped soluble leishmanial antigen induces antigen-specific T-helper type 1 immune response in BALB/c mice through uptake by peritoneal macrophages. Parasite Immunol. 2007;29:229-39.

10. Trif M, Guillen C, Vaughan DM, Telfer JM, Brewer JM, Roseanu A, et al. Liposomes as possible carriers for lactoferrin in the local treatment of inflammatory diseases. Exp Biol Med. 2001;226:559-64.

11. Benech R-O, Kheadr EE, Lacroix C, Fliss I. Antibacterial activities of nisin Z encapsulated in liposomes or produced in situ by mixed culture during cheddar cheese ripening. Appl Environ Microbiol. 2002;68:5607-19.

12. Laridi R, Kheadr EE, Benech R-O, Vuillemard JC, Lacroix C, Fliss I. Liposome encapsulated nisin Z: optimization, stability, and release during milk fermentation. Int Dairy J. 2003;13:325-36.

13. Cleveland J, Montville TJ, Nes IF, Chikindas ML. Bacteriocins: safe, natural antimicrobials for food preservation. Int J Food Microbiol. 2001;71:1-20.

14. Kuramitsu HK, He X, Lux R, Anderson MH, Shi W. Interspecies interactions within oral microbial communities. Microbiol Mol Biol Rev. 2007;71:653-70.

15. Nishikawara F, Katsumura S, Ando A, Tamaki Y, Nakamura Y, Sato K, et al. Correlation of cariogenic bacteria and dental caries in adults. J Oral Sci. 2006;48:245-51.

16. Selwitz RH, Ismail AI, Pitts NB. Dental caries. Lancet. 2007;369:51-9.

17. Paes Lame AF, Koo H, Bellato CM, Bedi G, Cury JA. The role of sucrose in cariogenic dental biofilm formation-new insight. J Dent Res. 2006;85:878-87.

18. Quivey Jr RG, Kuhnert WL, Hahn K. Genetics of acid adaptation in oral streptococci. Crit Rev Oral Biol Med. 2001;12:301-14.

19. Kreth J, Zhu L, Merritt J, Shi W, Qi F. Role of sucrose in the fitness of Streptococcus mutans. Oral Microbiol Immunol. 2008;23:213-9.

20. de Arauz LJ, Jozala AF, Mazzola PG, Penna TCV. Nisin biotechnological production and application: a review. Trends Food Sci Technol. 2009;20:146-54.

21. Willey JM, van der Donk WA. Lantibiotics: peptides of diverse structure and function. Annu Rev Microbiol. 2007;61:477-501.

22. Guerra NP, Pastrana L. Influence of $\mathrm{pH}$ drop on both nisin and pediocin production by Lactococcus lactis and Pediococcus acidilactici. Lett Appl Microbiol. 2003;37:51-5. 
23. McAuliffe O, Ross RP, Hill C. Lantibiotics: structure, biosynthesis and mode of action. FEMS Microbiol Rev. 2001;25:285-308.

24. Hsu STD, Breukink E, Tischenko E, Lutters MAG, de Kruijff B, Kaptein R, et al. The nisinlipid II complex reveals a pyrophosphate cage that provides a blueprint for novel antibiotics. Nat Struct Mol Biol. 2004;11:963-7.

25. Wiedemann I, Breukink E, van Kraaij C, Kuipers OP, Bierbaum G, de Kruijff B, et al. Specific binding of nisin to the peptidoglycan precursor lipid II combines pore formation and inhibition of cell wall biosynthesis for potent antibiotic activity. J Biol Chem. 2001;276:1772-9.

26. Hancock REW, Sahl H-G. Antimicrobial and host-defense peptides as new anti-infective therapeutic strategies. Nat Biotechnol. 2006;24:1551-7.

27. Jenssen H, Hamill P, Hancock REW. Peptide antimicrobial agents. Clin Microbiol Rev. 2006;19:491-511.

28. Were LM, Bruce BD, Davidson M, Weiss J. Size, stability, and entrapment efficiency of phospholipids nanocapsules containing polypeptide antimicrobials. J Agric Food Chem. 2003;51:8073-9.

29. Samuelson O, Haukland HH, Jenssen H, Kramer M, Sandvic K, Ulvatne H, et al. Induced resistance to the antimicrobial peptide lactoferricin B in Staphylococcus aureus. FEBS Lett. 2005;579:3421-6.

30. Kuwano K, Tanaka N, Shimizu T, Nagatoshi K, Nou S, Sonomoto K. Dual antibacterial mechanisms of nisin $\mathrm{Z}$ against gram-positive and gram-negative bacteria. Int $\mathrm{J}$ Antimicrob Agents. 2005;26:396-402.

31. Cotter PD, Hill C, Ross RP. Food microbiology: bacteriocins: developing innate immunity for food. Nat Rev Microbiol. 2005;3:777-88.

32. Akerey B, Le-Lay C, Fliss I, Subirade M, Rouabhia M. In vitro efficacy of nisin Z against Candida albicans adhesion and transition following contact with normal human gingival cells. J Appl Microbiol. 2009;107:1298-307.

33. Lian T, Ho RJY. Trends and developments in liposome drug delivery systems. J Pharm Sci. 2001;90:667-80.

34. Samad A, Sultana Y, Aqil M. Liposomal drug delivery systems: an update review. Curr Drug Deliv. 2007;4:297-305.

35. Hsieh YF, Chen TL, Wang YT, Chang JH, Chang HM. Properties of liposomes prepared with various lipids. J Food Sci. 2002;67:2808-13.

36. Khosravi-Darani K, Pardakhty A, Honarpisheh H, Rao VSNM, Mozafari MR. The role of high-resolution imaging in the evaluation of nanovesicles for bioactive encapsulation and targeted nanotherapy. Micron. 2007;38:804-18.

37. Shehata T, Ogawara K, Higaki K, Kimura T. Prolongation of residence time of liposome by surface-modification with mixture of hydrophilic polymers. Int J Pharm. 2008;359:272-9.

38. Yamauchi M, Tsutsumi K, Abe M, Uosaki Y, Nakakura M, Aoki N. Release of drugs from liposomes varies with particle size. Biol Pharm Bull. 2007;30:963-6.

39. Hemanth KM, Spandana V. Liposomal encapsulation technology: a novel drug delivery system designed for ayurvedic drug preparation. Int Res J Pharm. 2011;2:4-6.

40. Taylor TM, Bruce BD, Weiss J, Davidson PM. Listeria monocytogenes and Escherichia coli O157:H7 inhibition in vitro by liposome-encapsulated nisin and ethylenediaminetetraacetic acid. J Food Saf. 2008;28:183-97.

41. Hofheinz RD, Gnad-Vogt SU, Beyer U, Hochhaus A. Liposomal encapsulated anti-carrier drugs. Anticancer Drugs. 2005;16:691-707.

42. Johnston MJ, Semple SC, Klimuk SK, Ansell S, Maurer N, Cullis PR. Characterization of the drug retention and pharmacokinetic properties of liposomal nanoparticles containing dihydrosphingomyelin. Biochim Biophys Acta. 2007;1768:1121-7.

43. Ikehara Y, Niwa T, Biao L, Kabata-Ikehara S, Ohashi N, Kobayashi T, et al. A carbohydrate recognition-based drug delivery and controlled release system using interperitoneal macrophages as a cellular vehicle. Cancer Res. 2006;66:8740-8. 
44. Mozafari MR, Johnson C, Hatziantoniou S, Demetzos C. Nanoliposomes and their applications in food nanotechnology. J Liposome Res. 2008;18:309-27.

45. Taylor TM, Davidson PM, Bruce BD, Weiss J. Liposomal nanocapsules in food science and agriculture. Crit Rev Food Sci Nutr. 2005;45:587-605.

46. Matsui M, Shimizu Y, Kodera Y, Kondo E, Ikehara Y, Nakanishi H. Targeted delivery of oligomannose-coated liposome to the omental micrometastasis by peritoneal macrophages from patients with gastric cancer. Cancer Sci. 2010;101:1670-7.

47. Date AA, Joshi MD, Patravale VB. Polymeric parasitic disease: liposomes and polymeric nanoparticles versus lipid nanoparticles. Adv Drug Deliv Rev. 2007;59:505-21.

48. Ikegami S, Yamakami K, Ono T, Sato M, Suzuki S, Yoshimura I, et al. Targeting gene therapy for prostate cancer cells by liposomes complexed with anti-prostate-specific membrane antigen monoclonal antibody. Hum Gene Ther. 2006;17:997-1005.

49. Allen TM, Cullis PR. Drug delivery systems: entering the mainstream. Science. 2004;303:1818-22.

50. Kozako T, Hirata S, Shimizu Y, Satoh Y, Yoshimitsu M, White Y, et al. Oligomannose-coated liposomes efficiently induce human T-cell leukemia virus-1-specific cytotoxic T lymphocytes without adjuvant. FEBS J. 2011;278:1358-66.

51. Koning GA, Eggermont AMM, Lindner LH, ten Hagen TLM. Hyperthemia and thermosensitive liposomes for improved delivery of chemotherapeutic drugs to solid tumors. Pharm Res. 2010;27:1750-4.

52. Garbuzenko OB, Saad M, Pozharov VP, Reuhl KR, Mainelis G, Minko T. Inhibition of lung tumor growth by complex pulmonary delivery of drugs with oligonucleotides as suppressors of cellular resistance. Proc Natl Acad Sci U S A. 2010;107:10737-42.

53. Akbarzadeh A, Rezaei-Sadabady R, Davaran S, Joo SW, Zarghami N, Hanifehpour Y, et al. Liposome: classification, preparation, and applications. Nanoscale Res Lett. 2013;8:102-10.

54. Allon N, Saxena A, Chambers C, Doctor BP. A new liposome-based gene delivery system targeting lung epithelial cells using endothelin antagonist. J Control Release. 2010;160:217-24.

55. Volodkin DV, Ball V, Voegel JC, Mohwald H, Dimova R, Marchi-Artzner V. Control of the interaction between membranes or vesicles: adhesion, fusion and release of dyes. Colloids Surf A Physicochem Eng Asp. 2007;303:89-96.

56. Simoes S, Moreira JN, Fonseca C, Duzgunes N, de Lima MC. On the formulation of pH-sensitive liposomes with long circulation times. Adv Drug Deliv Rev. 2004;56:947-65.

57. Barenholz Y. Liposome application: problems and prospects. Curr Opin Colloid Interface Sci. 2001;6:66-77.

58. Bhardwaj U, Burgess DJ. Physicochemical properties of extruded and non-extruded liposomes containing the hydrophobic drug dexamethasone. Int J Pharm. 2010;388:181-9.

59. Daghastani KRP, Ferreira RB, Thedei GJ, Maggio B, Ciancaglini P. Lipid compositiondependent incorporation of multiple membrane proteins into liposomes. Colloids Surf B. 2004;36:127-37.

60. Anderson M, Omri A. The effect of different lipid components on the in vitro stability and release kinetics of liposome formulations. Drug Deliv. 2004;11:33-9.

61. Were LM, Bruce B, Davidson PM, Weiss J. Encapsulation of nisin and lysozyme in liposomes enhances efficacy against Listeria monocytogenes. J Food Prot. 2004;67:922-7.

62. Dhoot NO, Wheatley MA. Microencapsulated liposomes in controlled drug delivery: strategies to modulate drug release and eliminate the burst effect. J Pharm Sci. 2003;92:679-89.

63. Malheiros PS, Daroit DJ, Brandelli A. Food applications of liposome-encapsulated antimicrobial peptides. Trends Food Sci Technol. 2010;21:284-92.

64. Lu T, Wang Z, Ma Y, Zhang Y, Chen T. Influence of polymer size, liposomal composition, surface charge, and temperature on the permeability of $\mathrm{pH}$-sensitive liposomes containing lipid-anchored poly(2-ethylacrylic acid). Int J Nanomedicine. 2012;7:4917-26. 
65. Taylor TM, Gaysinsky S, Davidson PM, Bruce BD, Weiss J. Characterization of antimicrobial-bearing liposomes by $\zeta$-potential, vesicle size, and encapsulation efficacy. Food Biophys. 2007;2:1-9.

66. Takakura N, Wakabayashi H, Ishibashi H, Teraguchi S, Tamura Y, Yamaguchi H, et al. Oral lactoferrin treatment of experimental oral candidiasis in mice. Antimicrob Agents Chemother. 2003;47:2619-23.

67. Kisel MA, Kulik LN, Tsybovsky IS, Vlasov AP, Vorob'yov MS, Kholodova EA, et al. Liposomes with phosphatidylethanol as a carrier for oral delivery of insulin: studies in the rat. Int J Pharm. 2001;216:105-14.

68. Hayashida K, Kaneko T, Takeuchi T, Shimizu H, Ando K, Harada E. Oral administration of lactoferrin inhibits inflammation and nociception in rat adjuvant-induced arthritis. J Vet Med Sci. 2004;66:149-54.

69. Ishikado A, Imanaka H, Takeuchi T, Harada E, Makino T. Liposomalization of lactoferrin enhanced it's anti-inflammatory effects via oral administration. Biol Pharm Bull. 2005;28:1717-21.

70. Teixeira ML, dos Santos J, Silveria NP, Brandelli A. Phospholipid nanovesicles containing a bacteriocin-like substance for control of Listeria monocytogenes. Innov Food Sci Emerg Technol. 2008;9:49-53.

71. Breukink E, Ganz P, de Kruijff B, Seelig J. Binding of nisin Z to bilayer vesicles as determined with isothermal titration calorimetry. Biochemistry. 2000;39:10247-54.

72. Deegan LH, Cotter PD, Hill C, Ross P. Bacteriocins: biological tools for bio-preservation and shelf-life extension. Int Dairy J. 2006;16:1058-71.

73. Mugabe C, Halwani M, Azghani AO, Lafrenie PM, Omri A. Mechanisum of enhanced activity of liposome-encapsulated aminoglycosides against resistant strains of Pseudomonas aeruginosa. Antimicrobial Agents Chemother. 2006;50:2016-22.

74. Branen JK, Davidson PM. Environment of nisin, lysozyme, and monolaurin antimicrobial activities by ethylenediaminetetraacetic acid and lactoferrin. Int $\mathrm{J}$ Food Microbiol. 2004;90:63-74. 